Portland State University

PDXScholar

Ready? Set? Go?: Examining Organizational

Readiness for Change in a Quality Improvement Intervention for Youth Mentoring Programs

\author{
Renee Spencer \\ Boston University \\ Alison L. Drew \\ Boston University \\ Carla Herrera \\ Bowen McBeath \\ Portland State University, mcbeath@pdx.edu \\ Thomas E. Keller \\ Portland State University, kellert@pdx.edu
}

Follow this and additional works at: https://pdxscholar.library.pdx.edu/socwork_fac

Part of the Social Work Commons

Let us know how access to this document benefits you.

\author{
Citation Details \\ Spencer, R., Drew, A. L., Herrera, C., McBeath, B., \& Keller, T. E. (2021). Ready? Set? Go?: Examining \\ Organizational Readiness for Change in a Quality Improvement Intervention for Youth Mentoring \\ Programs. Journal of the Society for Social Work and Research.
}

This Post-Print is brought to you for free and open access. It has been accepted for inclusion in School of Social Work Faculty Publications and Presentations by an authorized administrator of PDXScholar. Please contact us if we can make this document more accessible: pdxscholar@pdx.edu. 


\section{Ready? Set? Go?: Examining Organizational Readiness for Change in a Quality Improvement Intervention for Youth Mentoring Programs}

Renee Spencer, Boston University

Alison L. Drew

Carla Herrera

Bowen McBeath

Thomas E. Keller 


\begin{abstract}
The number of youth mentoring programs has risen significantly in recent decades. This trend, coupled with evidence that programs employing a greater number of empirically supported practices achieve more positive effects for youth participants, has prompted increasing interest in how to promote more widespread use of evidence-based practice standards in mentoring programs. In an effort to describe and better understand efforts to implement recommended standards, we studied a multi-level initiative sponsored by a national advocacy organization in which its state-level Affiliates guided local mentoring programs through a structured quality improvement process. Specifically, we examined organizational readiness for change among mentoring program staff as well as among staff from the state-level Affiliates charged with supporting the mentoring programs as they implemented their change process. Analysis of indepth qualitative interviews with 44 staff indicated that programs and supporting Affiliates were generally highly motivated and committed to the change process. However, because this enthusiasm was not always accompanied by the resources needed to make program change, staff were more measured in their perceptions of change efficacy and noted corresponding challenges that weakened the implementation process. Implications of these findings for practice and future research are discussed.
\end{abstract}

Keywords: youth mentoring, evidence-based practice, quality rating and improvement systems, organizational readiness for change, program quality improvement, qualitative methods 
Mentoring as an intervention to support youth development has grown in popularity in recent decades (Rhodes \& DuBois, 2008), and an estimated 2.5 million adults in the U.S. volunteer to serve as mentors through formal youth-serving organizations (Raposa et al., 2017). Youth mentoring programs vary on multiple dimensions, such as their objectives, models, and populations served (Karcher et al., 2006), yet meta-analytic reviews demonstrate the overall effectiveness of mentoring in enhancing youth outcomes across a number of social, developmental, behavioral, and academic domains (DuBois et al., 2002; DuBois et al., 2011; Raposa et al., 2019; Tolan et al., 2014). Evidence further indicates that programs employing a greater number of empirically supported practices promote longer-lasting mentoring relationships (Kupersmidt et al., 2017) and attain more positive effects for youth participants (DuBois et al., 2002). Consequently, there is an emphasis on improving mentoring program effectiveness by identifying best practices in these programs (Komosa-Hawkins, 2009; Miller, 2007; Sipe, 2002) and supporting their implementation through organizational improvement initiatives and the professional development of program staff (Stoeger et al., 2021; Wandersman et al., 2006). Focusing on a quality improvement initiative for youth mentoring programs, the current study investigates key preconditions for its effective implementation by examining factors associated with organizational readiness for change, specifically change motivation and change efficacy (Weiner, 2009).

\section{Quality Improvement in Youth Programs}

Youth mentoring practice and research mirrors trends and findings for youth development programs more broadly. Research indicates higher quality implementation of youth program services is associated with better youth outcomes (Cross et al, 2010; Durlak \& Dupre, 2008), and a long-standing priority has been to better understand, assess, and improve the quality 
of youth afterschool programs (Granger, 2010; Smith \& Akiva, 2008; Yohalem et al., 2009). As a result, observational tools to assess the quality of youth program settings and point-of-service interactions have been developed as the basis for encouraging staff to form learning communities that focus on areas of program improvement and accountability (Smith et al., 2009; Yohalem \& Wilson-Ahlstrom, 2010). Youth program quality improvement efforts typically involve first using such an assessment to evaluate program features and practices, then reviewing the assessment data to establish improvement goals, and finally engaging staff in targeted training and technical assistance to enhance performance (Smith et al., 2006; Spielberger et al., 2009; Yohalem et al., 2009).

As an example, a project to increase quality in afterschool programs employed regular observation and coaching of staff, ongoing staff training, and the use of data to measure progress (Sheldon et al., 2010). In a rare large-scale experimental study, managers of 87 afterschool programs were trained to carry out a quality improvement process that included assessment and data-driven planning at the organizational level followed by coaching focused on improving individual staff performance in point-of-service interactions with youth. This research indicated a positive impact of the intervention on the improvement focus of managers, the adoption of improvement practices by managers and staff, and ultimately the quality of instruction delivered by staff (Smith, et al., 2012).

These strategies for youth program quality improvement align with core elements of Quality Rating and Improvement Systems (QRIS) that are prevalent in the fields of childcare and early education (Goffin \& Barnett, 2015; Tout, 2013). QRIS is a systematic approach adopted in most states to assess, improve, and communicate the quality of service delivery in these sectors. The common features of QRIS include: a) established quality standards; b) an assessment 
process for assigning ratings based on those standards; c) a process for supporting providers in quality improvement; d) incentives for improvement; and e) dissemination of quality indicators to consumers, such as parents (Boller et al., 2015; Mitchell, 2005; Zellman \& Perlman, 2008). QRIS in child care and early education began as a market-based strategy to promote program quality, but it also has become an avenue for systems change by providing a delivery mechanism for professional development and an accountability tool for program outcomes (Goffin \& Barnett, 2015). Despite the widespread investment of state policy-makers and administrators in QRIS, very little research has investigated the scope of adoption or the factors that influence the voluntary participation of providers in the QRIS process (Jenkins et al., 2021) or considered the perspective of providers engaging in QRIS (Hallam et al., 2017).

Similarly, there has been relatively little attention to the circumstances under which quality improvement initiatives for youth development programs have been implemented. For instance, staff may consider external evaluation processes and technical support to be hierarchically imposed and incongruent with the realities of their programs or beyond their capacity to implement given resource constraints (Baldwin \& Wilder, 2014). Likewise, a wide variety of interrelated structural factors (e.g., number of youth served, staffing ratios, facilities, leadership experience) can inhibit program capacity to effectively engage in quality improvement protocols — particularly protocols that lack flexibility and appropriate fit (Baldwin et al., 2015). Attention also must be given to the skills and perspectives of program leaders, who are critical in balancing a complex set of demands in their efforts to achieve and maintain youth program quality (Larson \& Walker, 2010). In one of the few process-oriented studies to investigate how a youth program quality improvement initiative actually operated, the authors note, "Among other findings, we discovered that quality improvement begins with program 
managers, who then lead the process of change" (Devaney et al., 2012, p. 2). These authors found that, to make meaningful progress in enhancing program quality, managers needed to focus on structural changes (refining policies and procedures, shifting job duties), organizational climate (better communication, greater alignment and intentionality) and their own supervision and management (providing more training and feedback).

\section{Organizational Readiness for Change}

Implementing organizational-level change is difficult, even among those highly invested in change efforts (Aarons et al., 2011; Holt \& Vardaman, 2013). The challenges of implementing change are especially notable in human service organizations due to the complexities inherent in the delivery of these kinds of services (McBeath \& Hopkins, 2019). The extensive literature on organizational change and implementation of evidence-based practices has emphasized the importance of several intra- and inter-organizational factors in the success of the implementation process (e.g., Aarons et al., 2011; Moullin et al., 2019).

For example, being involved in a network of programs is an important interorganizational factor that supports implementation and change efforts (Lengnick-Hall et al., 2021).

Interorganizational networks can act as knowledge networks for supporting the dissemination and implementation of effective practices and quality improvement processes (Phelps et al., 2011). Further, within organizations, a shared commitment to common goals can foster an environment in which staff view the adoption of certain practices as having a positive impact on both the clients served and the organization more broadly (Farahnak et al., 2020).

However, even if programs value and strive to achieve evidence-based quality standards, they must be prepared to make changes required for implementation of those practices. The evolving literature on implementation science consistently emphasizes organizational readiness 
for change (“ORC”) as critical to successful change efforts (Holt \& Vardaman, 2013; Weiner et al., 2020). Organizational readiness for change reflects the "extent to which organizational members are psychologically and behaviorally prepared to implement organizational change” (Weiner et al., 2008, p. 381). According to Weiner (2009), the two main components of ORC are change commitment and change efficacy. Commitment refers to the organizational members' shared investment in the change and dedication to taking needed action. Commitment can be based on valuing the change, feeling compelled to make the change, or feeling obligated to do so. Change efficacy is multi-faceted, encompassing the organizational members' shared belief in their capability to complete the tasks associated with the change, the availability of needed resources, and the receptivity of the context for the desired change.

Weiner's theory emphasizes collective attitudes within the organization and notes the differential weight of particular members (e.g., management, administration, service providers, supervisors, clients, board members). The theory also highlights the role that people's perceptions of their abilities play in the change process. When organizations have high levels of ORC, "members are more likely to initiate change, exert greater effort, exhibit greater persistence, and display more cooperative behavior," which results in "more effective implementation" (Weiner, 2009, p. 1). In this way, the framework sheds light on how an organization may value implementing evidence-based practices, but not succeed in their efforts to do so. The ORC theory's focus on the attitudes and beliefs of key personnel makes it particularly well suited for the present study, which relies on the perspectives and experiences of those responsible for undertaking a quality improvement process.

\section{Quality Mentoring System Initiative}


Quality improvement initiatives in the field of youth mentoring have been promoted by MENTOR, the leading national advocacy organization for youth mentoring programs in the United States. MENTOR is the parent organization for a network of state-level Affiliates (e.g., Massachusetts Mentoring Partnership, MENTOR Minnesota) that serve mentoring programs in their states. MENTOR and its Affiliates provide infrastructure and resources to benefit the spectrum of youth mentoring programs. Specifically, MENTOR and its affiliates operate as intermediaries to support program service providers by disseminating best practices, providing training and technical assistance, advocating for mentoring, and raising public awareness of mentoring. As part of these efforts, MENTOR has invested heavily in the Elements of Effective Practice for Mentoring (“EEPM"; Garringer et al., 2015) — now in its fourth iteration —a widely distributed compendium of research-informed practice standards for mentoring programs. These standards relate to the practice of fostering mentoring relationships, such as procedures for recruiting, screening, training, and matching program participants and supporting the development of their mentoring relationships. In addition, the EEPM addresses the organizational and operational practices needed to build and sustain effective mentoring programs, with guidelines in areas such as governance, finance, personnel management, stakeholder engagement, and program evaluation.

Despite MENTOR's efforts to promulgate program standards, research-supported practices have been unevenly implemented in the mentoring field (Garringer, et al., 2017; Kupersmidt et al., 2017). A recent national survey of youth mentoring programs found that nearly half were not even aware of the EEPM. Even among programs aware of the EEPM, efforts toward actually implementing the recommended practices were mixed (Garringer et al., 2017). Despite notable enthusiasm for implementation of best practices in the field of youth 
mentoring (Stoeger et al., 2021), will without readiness may partially explain why successive meta-analyses of mentoring program evaluations have shown only negligible improvement in the average magnitude of effects for youth mentoring programs over the past two decades (DuBois et al., 2011; DuBois et al., 2002; Raposa et al., 2019). Even among programs aware of best practices, uptake may be limited if programs stumble in their efforts to implement these practices.

To encourage greater use of EEPM practices, two of MENTOR's state-level Affiliates developed interventions incorporating the core elements of quality rating and improvement systems for working with mentoring programs to improve their practices. The two interventions, collectively labeled the Quality Mentoring System (QMS), were similar in their application of QRIS methods. In both cases, the Affiliates: a) guided local mentoring programs through a selfassessment process based on adherence to the $3^{\text {rd }}$ Edition of the Elements of Effective Practice for Mentoring (MENTOR, 2009); b) offered advice and consultation in developing a tailored workplan to achieve higher standards; and c) provided training and technical assistance to help programs accomplish their improvement goals over the course of approximately one year. The primary distinction between the two QMS models was in how they structured incentives and shared assessment data with stakeholders. After a re-evaluation to document progress after one year, one model provided a "badge" to indicate when a certain number of standards had been achieved. The other model endorsed a continuous improvement philosophy and indicated that participating programs demonstrated an ongoing commitment to quality.

Based on the positive experiences of the two Affiliates that pioneered QMS development and implementation (hereafter "expert Affiliates"), MENTOR organized a pilot initiative in which eight new Affiliates (hereafter "novice Affiliates") were selected to implement the next 
wave of QMS intervention with mentoring programs in their respective states. The eight novice Affiliates represented approximately one third of MENTOR's Affiliate network at the time. The two expert Affiliates that developed the QMS intervention were each assigned to advise and coach four of the novice Affiliates adopting the process. This simultaneous pilot implementation by the novice Affiliates was evaluated for both process and outcome. MENTOR has since integrated lessons from this pilot effort into a revised framework for a nationwide system of quality improvement reflecting the updated standards of the $4^{\text {th }}$ Edition of the EEPM (Garringer et al., 2015).

\section{Current Study}

The QMS pilot implementation represents the first time a QRIS approach has been evaluated in the field of youth mentoring and one of the few times such an approach has been investigated within the field of youth development. Consequently, the current study offers an opportunity to build knowledge regarding the organizational motivations and capacities that contribute to undertaking this type of quality improvement process. To describe and better understand the role of organizational readiness for change in a coordinated pilot initiative to promote the implementation of recommended best practices in youth mentoring, we examined perceptions of staff from the novice state-level Affiliates charged with supporting programs as they implemented their change process as well as the mentoring program staff engaging in the QMS process within their programs. For both the mentoring program staff and Affiliate staff, the following questions were addressed: (1) How did the mentoring program staff and the Affiliate staff describe their organization's commitment to the QMS change process?; (2) What were their perceptions of their organization's efficacy or ability to engage in the QMS process, and 
specifically what factors facilitated the organization being ready (or not) to make the changes required by QMS?

\section{Method}

The present study is part of a larger, multi-state, mixed methods randomized controlled trial and implementation study of QMS (authors, 2018). For the randomized trial, the eight novice Affiliates implementing QMS for the first time recruited mentoring programs in their respective service areas to participate in the intervention, and within each state these programs were randomly assigned to engage in QMS $(n=34)$ or to a waitlist control condition $(n=32)$. The current analysis examines organizational change commitment and change efficacy at two levels: among mentoring programs engaging in the QMS intervention condition; and among the eight novice state-level Affiliates tasked with guiding the programs in their QMS quality improvement efforts. In this implementation process, both the programs and the Affiliates were undergoing a change process. The programs were working to implement EEPM standards through the QMS process, and the novice Affiliates were new to supporting programs in implementing QMS.

\section{Participants}

The current study utilized qualitative interview data collected from staff representing the eight novice Affiliates leading the QMS implementation $(n=10)$ as well as staff $(n=34)$ representing 20 mentoring programs implementing QMS in the treatment condition of the study (Table 1). At each novice Affiliate, the staff person responsible for leading the QMS implementation process was invited to participate in an interview, conducted at least 15 months after the randomization of programs in their state and the beginning of QMS implementation. If the Affiliate staff member leading the QMS effort left their job before the 15-month mark, that 
staff person was interviewed at the time of departure, and their replacement also was interviewed at 15 months.

The interviewed mentoring program staff represented seven states, as one Affiliate withdrew from the study before any of its programs had been randomized and baseline data were collected. Purposeful sampling was used to identify 20 participating mentoring programs that reflected the diversity of the mentoring field in size, organizational structure (embedded in a community organization, stand-alone non-profit, other), program model (whether the program offered one-on-one, group, multi-mentor, and/or team mentoring relationships), and setting (urban, suburban, rural; see Table 2). Sampling was conducted to include 3 to 4 programs working with each state Affiliate. If an Affiliate served fewer than 3 treatment programs, all treatment programs were included in the sample. Affiliate staff helped identify the staff person responsible for leading QMS at each selected program. That person was invited by the research team to participate in an interview and was also asked to identify a second staff member who worked directly with mentors and mentees, would be familiar with QMS, and would be able to participate in an interview. In 12 programs, a second staff person was identified and invited to participate in an interview. In 10 mentoring programs, there was only one staff person or only one person who had knowledge of QMS implementation.

\section{Procedures}

A majority of participants completed a one-time, semi-structured, in-person interview during a site visit to their agency $(n=38)$. The remaining interviews $(n=6)$ were conducted by telephone. In accordance with an IRB-approved protocol, written consent was obtained prior to interviews, and participants received a $\$ 30$ gift card in recognition of their participation.

Interviews were conducted by the first and second authors. The first author is a professor of 
social work with expertise in youth mentoring research. At the time of the interviews, the second author was a PhD student under the supervision of the first author, with five years of professional experience working in the youth mentoring field. The interviewers were part of the independent research team and were not involved with implementing QMS. In the interviews, respondents reported on the experiences of their organizations in implementing QMS. Interview questions for both the novice Affiliates and the mentoring programs addressed how the organization learned about QMS, how buy-in was obtained, why the organization chose to do QMS, and challenges and facilitators in the implementation process. Interviews were audio-recorded and transcribed verbatim. Transcripts were verified for accuracy and de-identified, with all participant and program names replaced with study identification codes.

\section{Data Analysis}

Interviews were coded thematically (Braun \& Clarke, 2006). Two members of the research team familiar with the interviews developed an initial codebook based on the interview protocols, the larger study's central research questions, and previous research. The codebook included 9 sections (e.g., Decision to pursue QMS), each with relevant codes (e.g., Learned about QMS, Early expectations). The codebook was reviewed by members of the research team to ensure clarity. Interviews were coded by a team of 7 research assistants using NVivo. The codebook was reviewed throughout the coding process, and additional codes were added as they were identified. One team member served as the lead coder, training all coders and reviewing their work to ensure consistency in coding across interviews.

The two sections of the codebook that were relevant to the current research questions and their corresponding codes were included for analysis. The first section included "Decision to pursue QMS,” which addressed the novice Affiliate's or mentoring program's process of 
learning about and deciding to engage with QMS. The second section was "Steps in preparation for QMS," which addressed the steps that the novice Affiliate or mentoring program took to prepare for undertaking the QMS process. After coding was completed, a series of conceptually clustered matrices (Miles et al., 2014) were created. Initial matrices organized the quotes associated with the codes by interview. Participant quotes were read multiple times by a team of three coders, led by the second author, to identify themes. Once themes were identified at the participant level, the second author created a final matrix to organize and sort themes across participants. At this stage, the analysis was organized around Weiner's (2009) model of organizational readiness for change, specifically participants' perceptions of organizational change commitment and change efficacy. Thus, the ORC model was used to frame and structure the thematic organization of data already coded. The initial sorting of themes was discussed amongst the authors and revised as needed. Particular attention was paid to how well the themes corresponded with Weiner's model. All authors ultimately agreed that the model was an appropriate framework for understanding and presenting the data.

\section{Results}

\section{Commitment to the Change Process}

\section{Novice Affiliates}

Staff from the novice Affiliates reported a variety of reasons why they and/or their organizations valued and were committed to implementing QMS (see Table 3 for themes). First and foremost, many respondents felt that QMS aligned with, and appropriately expanded, the role of their Affiliate within their state's mentoring community. Importantly, Affiliate staff tended to understand the value of utilizing evidence-based standards and saw QMS as an opportunity to help mentoring programs in their region both demonstrate and improve their 
quality by increasing their adherence to these practices. Several Affiliate staff noted that taking on this role would improve the sustainability of the affiliate itself by providing a clear added value to the field. As one staff noted, prior to implementing QMS, the Affiliate had to rely on activities like counting the number of trainings they had conducted, which was unsatisfactory because, "That's not knowing whether you moved the mark at all." QMS enabled the Affiliate to "really dig in," as it could document that a program had "changed X amount because they've done these different kinds of things and implemented them in their work plan.”

In some cases, novice Affiliates had already been looking for ways to engage in a QMSlike process. Some had even considered developing a quality improvement system of their own prior to having the opportunity to participate in the pilot project. Affiliate staff generally reported their appreciation for not needing to invest the time and money to create something new when these systems already had been developed. As one staff person explained, hearing about the QMS initiative piqued interest in the QMS tools: "we knew that [expert Affiliate] had this assessment that they were using that we really wanted to get our hands on because our process of determining if a program was meeting evidence-based standards... was... laborious.”

Commitment from stakeholders across the organization was seen as crucial for successfully delivering QMS. Despite high levels of personal commitment reported by most respondents, some noted challenges in getting buy-in from key parties such as the Affiliate's leadership team or board of directors. Often, those who were initially interested in QMS were tasked with helping others understand the value and purpose of engaging in the QMS process:

It took some time for us to get our Board on [board]... because for a long time, we had been doing the same work... We were known as the mentor training organization, and we provided training and technical assistance... This quality process was totally new work... It did take some time for our Board to understand why and how, we [would] do that work. 
In most cases, respondents reported that these key Affiliate stakeholders eventually understood the value of QMS and provided necessary supports. However, in one case, the leadership team agreed to allow the Affiliate to engage in QMS but was never really committed to the change, which resulted in a lack of resources and undermining the work of the staff person involved in implementing QMS.

\section{Mentoring Programs}

Overall, mentoring program staff reported a strong organizational commitment to engaging in the QMS process due to a wide variety of contributing factors. As with Affiliates, mentoring program participants indicated that their programs found QMS to be an important way to increase their implementation of recommended practices or "industry standards." As one program staff explained, "I knew there was the focus on the evidence-based... practices... [QMS] reinforced that we were doing those things, or areas we needed to work on, or whatever. I thought that couldn't hurt." Programs saw QMS as a way to enhance and even expand their program, demonstrate their impact, and, for newer programs, to catch up with other programs in their region.

Many mentoring programs also noted that they valued the "proof of quality" offered by QMS. The Affiliates offered mentoring programs a variety of ways to share publicly that they were high-quality programs or engaged in quality improvement. These markers of quality included an email badge, improved presence on the Affiliate's website, or achievement of different levels of "membership." Program staff expected that these proofs would be especially appealing to funders looking to make smart investments based on the quality of a program's practices. Some also indicated that they believed the public displays regarding quality would help recruit volunteer mentors trying to identify effective programs. 
The structure of the QMS intervention itself was also appealing to these mentoring programs. Many program staff noted appreciating that the Affiliate staff member was someone outside their own organization, which would enable them to get unbiased feedback. Many further emphasized that their program had a good relationship with the Affiliate, which helped them trust that QMS was a valuable investment and also feel confident that the Affiliate would provide high-quality support throughout their involvement. As one staff person explained:

We became partners with [Affiliate]... connecting with them in a very positive way. They asked if we would be interested in doing the QMS, and I had heard about the QMS through some of the things that were going on in the state, and said "Yes. I'd be very interested..." An outside evaluation is always a very good thing to do.

Program staff also reported their programs found the self-assessment process appealing as it would gave their staff time to take a close look at their practices and set their own goals for improvement.

As was true with Affiliates, there were typically many stakeholders, including the executive director and leadership team, who ultimately needed to be committed to making change through the QMS process. In addition, approval was typically required from the program's board of directors, the district office for school-run programs, or a national office for some nationally affiliated programs. In general, program staff indicated that getting stakeholder buy-in was a relatively easy process, as QMS's purpose was straightforward: “Getting Board buy-in was super easy ... They were excited about it ... They understood what it needed ... And how it could benefit us."

Yet, in some programs, staff felt they had no choice regarding QMS participation. In some cases, this was the view of a staff person who had been hired after the program had agreed to begin QMS, making the work part of that person's job description. Two Affiliates required their programs to participate in the QMS process to be eligible to access ongoing resources from 
the Affiliate, including funding. Similarly, one respondent explained that his school-based mentoring program was chosen by the district that oversees the program to complete QMS without consulting the staff who run the program: "It was one of those .... 'volun-told' type deals. Because we were programming the [neighborhood] which traditionally has the most challenges behaviorally, academically, all that... the [school district] selected us to be a part of that study." Some staff who felt they lacked choice about participating in QMS reported a lower level of commitment to QMS, especially if they also did not understand the potential benefits of their involvement.

\section{Change Efficacy: Perceptions of Organizational Preparedness and Resources}

\section{Novice Affiliates}

Overall, novice Affiliate staff reported an extensive planning process leading up to implementing QMS with the goal of making sure that there was a clear understanding of what tasks, personnel, and resources were required for QMS and how implementation would roll out. Some Affiliates reported that they had explored multiple options for quality improvement, including other processes like QMS. Other Affiliates were heavily involved in the national planning for the QMS initiative, including preparation for the pilot initiative and evaluation. As one respondent explained, "For maybe 2 years before [starting QMS], I had gone back to DC to attend meetings... chaired a committee, on... cost and staffing for [Affiliates] to take this on." While most Affiliates were highly engaged in exploring the task demand for QMS implementation, a few were less prepared, particularly those added to the pilot as it was beginning. These Affiliates reported not being given enough information to really understand what QMS would require of them. 
Study participants identified key resources they thought were required for Affiliates to feel confident in their ability to implement QMS. Important internal resources included sufficient staff with the expertise to carry out implementation (and retention of those staff), funding specifically dedicated to QMS, and appropriate technology for organizing and managing documents and communication with mentoring program staff (e.g., database, video conferencing, cloud storage). In preparation for QMS, some Affiliates said they had planned changes to their staffing to accommodate the increased workload. A respondent from one Affiliate, which was embedded in a larger organization, explained how following through on the changes that were planned in preparation for QMS was challenging at times: "Our intent was... fundraising... that would allow us to increase our capacity by at least 1 , if not 2 , people" in order to manage the QMS process. However, "a lot of that got pushed aside as our [lead agency] went through some shrinking in other ways." Challenges and delays were also reported as occurring when a new staff person was in charge of leading QMS, as was the case in two Affiliates where the first QMS lead left the agency as well as in another Affiliate where a new staff person was hired to manage QMS at the beginning of implementation. These transitions were particularly challenging when experienced staff were not consistently available to train the new person.

Affiliates indicated that they relied on support from external resources to improve their ability to implement QMS beyond what they could accomplish on their own. First and foremost, the novice Affiliates emphasized that they expected their expert Affiliates would be important resources to help provide training, materials (e.g., templates), timelines and best practices around QMS implementation. In addition, some respondents talked about the importance of participating in group calls with staff from other Affiliates implementing QMS — a process that facilitated sharing around practices and helped newer staff gain an understanding of QMS more quickly. 
Further, staff noted that not all novice Affiliates had existing resources to offer their programs on all aspects of quality programming (e.g., financial management, research and evaluation). These Affiliates used external resources including their expert Affiliate and local non-profit experts to supplement their own expertise.

Finally, situational factors, specific to the Affiliate, sometimes influenced the Affiliate's sense of preparedness to engage in the QMS process. Salient in these interviews was how having the right person leading QMS implementation at the Affiliate influenced their readiness and ability to take on QMS. For example, despite a lack of strong support from the leadership team and insufficient resources and time, one staff person had a relatively easy time preparing for QMS because the respondent was willing ("I was the zealot!) and skilled ("I have an evaluation frame of mind") enough to make change happen.

Affiliates noted that the critical role of staff in implementing QMS also meant that changes in Affiliate personnel during QMS implementation were difficult and often left a new staff person doubting their ability to follow through with QMS. As one respondent explained,

I had no overlap with [my predecessor]. She was [the] only person that did all this [QMS work]... So there really was no training .... except for when [she] came in [and] she met with me twice for two hours. So basically, [I] just read everything I possibly could... [My predecessor] had everything so well laid out... she's meticulous about... just having all the information that you needed. So I just read everything I could, and then I start[ed] talking to programs and .... that's kinda how I muddled through the process.

Unfortunately, this gap in QMS leadership was felt by the mentoring programs that had no contact or support from the Affiliate during the months when no one was leading QMS and during the time it took the new Affiliate staff to be on-boarded. While many local mentoring programs had strong relationships with the first QMS lead, it was noticeably harder for this new person to come in and confidently take on implementation of QMS. 


\section{Mentoring Programs}

Many mentoring program staff indicated that in hindsight they felt they did not have a clear understanding of what was expected of the program during the QMS process when they agreed to participate. Many reported that they were not fully aware of the steps required for QMS, the time it would take to complete tasks, or the breadth of the workload. In most of these cases, mentoring program staff felt their Affiliate did not provide enough or accurate enough information, which was assumed to be due to lack of experience with the QMS process rather than intentional deception. In a few cases, prior to starting QMS, the mentoring program expected that they were closer to meeting the practice standards outlined in QMS than they actually were, which resulted in a much larger workload after completing the self-assessment than they had planned. As one program staff person explained:

When we signed up for it, we didn't really understand how much work it was actually going to be... We just were kind of like, "Oh this will be cool." And then we started going through it. We're like, "Wow. This is like a full-time job to work through all of this."

Some programs, however, did report that their assessment of what was expected at the beginning turned out to be realistic and allowed them to be successful in meeting expectations:

[We're] a smaller program with one person... We'd only been [operating]... the program, for three years... If it would have been super intensive, it might not have been a good fit just for capacity reasons. But ... they assured us. They said it wouldn't be super time intensive.

As with Affiliates, mentoring programs reported requiring significant resources to prepare for successful QMS implementation, particularly human and financial resources. Mentoring programs needed sufficient staff time dedicated to the QMS process to make progress within the timeline set by the Affiliate. However, many programs experienced staffing challenges due to having few staff and high turnover. In addition, a few programs had more temporary staffing situations such as relying on AmeriCorps members, who lacked expertise and 
were term limited. Other mentoring programs were run completely by volunteers, who had limited time to devote to QMS. Participants in mentoring programs that reported having stable staff with strong understanding of evidence-based standards and experience with quality improvement also expressed greater confidence in their capacity for change.

In addition, mentoring program staff said they valued the resources and expertise offered by the Affiliate as a part of their QMS involvement, including the QMS materials themselves. Programs felt confident that their Affiliate would be available to support them and provide technical assistance and coaching as needed. Some programs had long been interested in making the improvements required by QMS, but admittedly had not known how to proceed without outside guidance and expertise. As one mentoring program staff person explained:

We don't like to reinvent the wheel... We wanna know where to improve and what we can do better... We knew [the Affiliate] would be honest about that and help us. Not just tell us... what we're doing wrong, but give us examples on how we can change it or grow.

There were specific situational factors noted by program staff that seemed to affect a mentoring program's confidence in its ability to implement QMS. The timing and pace of the QMS procedures, influenced in large part by the parameters of participating in a study, were a challenge for many programs. For example, some school-based mentoring programs require significant staff focus in late-summer and early-fall to recruit and match participants. These programs faced challenges when major QMS tasks needed to be done during these times of the school year. Similarly, some programs had very few staff, who consequently took on many roles within the organization. When these programs had major events, such as an annual fundraiser or match event, staff would be pulled away from QMS. 
Beyond timing, some mentoring program staff reported challenges within the organization that made implementing QMS difficult. Respondents indicated optimism regarding QMS implementation when their mentoring program had a strong culture of learning. However, some programs were more "stuck in their ways" and were more resistant to change. Similarly, some staff reported not having the power to actually make change within their mentoring program. One example of this was a nationally affiliated program that felt that the national office needed to lead program change efforts rather than individual programs:

If you decide you want to change your youth outcomes or mentor outcomes or [administer] some sort of survey.... With [National], you can't change those, so anything you want to do different, you have to add, you would add on versus replacing. I think that's where it becomes a pain.

\section{Discussion}

The experiences of the participants in this study illuminate the challenges faced by both the mentoring programs seeking to implement standards delineated in the Elements of Effective Practice for Mentoring (Garringer et al., 2015) and the state-level Affiliates tasked with supporting these programs in their efforts. Weiner's (2009) conceptualization of change commitment and change efficacy as important dimensions for organizational readiness for change was used to guide analysis of the participants' interviews. Results indicated that while many of these organizations may have had a high level of commitment to the QMS process, their commitment was not always accompanied by the strong change efficacy needed to make the changes. Both components of readiness were viewed as having contributed to implementation quality by both the mentoring programs and state Affiliates.

Most novice Affiliates and programs were generally highly committed to QMS, in part because the initiative aligned with existing organizational goals and would increase sustainability and growth and would validate the program. In those few cases where staff were not able to 
choose whether they were involved or did not understand the potential benefits of their involvement, staff reported less commitment to QMS. Commitment to the QMS process was critical at all levels, especially among those staff who were implementing the intervention, but also at all levels of leadership. Programs and novice Affiliates sometimes needed an internal champion who could "sell” QMS to leadership. Without consistent organizational commitment, individual staff struggled to implement QMS.

Important for change efficacy was strong, stable, and sufficient staffing at both the program and Affiliate levels. Furthermore, participants noted that Affiliate support was most helpful to programs in the context of long-standing relationships in which Affiliate staff were consistent and trusted. Programs with a strong "learning culture" that bought into the idea of change went into implementation more optimistically and found it easier to make program improvements. It was also important for staff to understand the potential demands and timeline of QMS prior to implementation and, at the Affiliate level, the types of resources that would be needed to successfully guide programs through implementation. Programs that found themselves being asked to do work that was different or more time intensive than they expected struggled with implementation.

In reflecting on the QMS process itself, mentoring program staff indicated that the structure of the intervention and the support provided by the Affiliates were critical to the programs achieving the desired changes. QMS offered a scaffolded process for programs to assess their needs for improvement, select and prioritize changes, develop a workplan, and obtain guidance, assistance, and resources to implement new policies and practices. Program staff described this approach as an appealing way to align practices with the program's own goals. 
Participating in QMS was described as having served as a catalyst for shifting quality improvement intentions into action.

The findings of this study align with themes in the organizational change literature highlighting that support for implementation processes matters greatly for achieving the desired outcomes (Holt \& Vardaman, 2013). QMS, a quality improvement intervention based on QRIS principles, has general acceptability among two important constituencies: staff in the intermediary organizations that oversee the process as well as staff in youth mentoring programs that want to enhance their services. This finding is consistent with the continuing expansion and evolution of the QRIS approach in other fields of practice, such as early education and childcare (Cannon et al, 2017). Importantly, the current study suggests potential receptivity to employing QRIS approaches developed for quality improvement in the youth development arena as well (e.g., Smith et al., 2006; Yohalem \& Alstrom-Winston, 2010).

The findings also support Weiner's theory (2009) that both commitment and efficacy matter for organizational readiness for change and suggest that cultivating both can prepare novice Affiliate and mentoring program staff to implement QMS processes in the ways necessary to be successful. Such conclusions are consistent with research showing that various facets of organizational readiness for change are associated with how successfully new innovations are adopted (Kelly et al, 2017). Interestingly, this research on organizational readiness for change indicates motivation is high when staff have identified program deficits and specific needs, which corresponds to the first step in the QMS process-program selfassessment. Furthermore, program resources and organizational climate predict the successful adoption of new practices, suggesting the importance of factors such as leadership, staff workloads, and financial resources (Kelly et al, 2017). Also, as found in prior ORC research 
(Datallo et al., 2017; Sanclimenti \& Caceda-Castro, 2017), being involved in a network was identified as a key factor in supporting QMS implementation as expert Affiliates shared resources and expertise and group calls among novice Affiliate staff encouraged sharing and learning.

In the current study, for example, program and novice Affiliate staff emphasized the importance of obtaining support for QMS from organizational leaders, which is consistent with research indicating that perceived leadership support enhances commitment to an organizational change initiative (Ford et al, 2021). Another important factor highlighted by the current findings was the disruption and difficulty caused by staff turnover, which has long been an issue in the field of youth mentoring (Keller, 2007). Although the loss of staff may derail or delay program change efforts, research suggests that implementation of evidence-based practices, and even fidelity monitoring, does not result in greater staff turnover but instead promotes greater staff retention (Aarons et al., 2009). Staff in the current study also cited the value of a culture of organizational learning in facilitating the QMS process, which aligns with frameworks linking organizational learning with implementation of evidence-based practices (Maynard, 2010). Undertaking a quality improvement or organizational change initiative also can support the development of an organizational learning orientation (Kerman et al, 2012).

\section{Limitations}

These considerations should be understood in relation to three study limitations. First, study findings were based on retrospective reports of organizational readiness for change. The participants were reflecting on the change process after completion of the intervention rather than reporting on their experiences as they were headed into the change process. As such, their reports may have been influenced by their experiences with the QMS process (e.g., those who 
had an easy time implementing QMS may report having been more prepared). Second, although we sampled participants from a high percentage of the programs that engaged in the QMS process, the overall sample remains relatively small. It is also important to note that the programs that engaged in QMS were recruited by the Affiliates and as such were typically ones with whom the Affiliates already had some type of relationship. More effort may be required to engage and prepare programs that do not already have an established relationship with a local Affiliate.

Finally, participants' descriptions of the burdens associated with QMS may have been influenced by their participation in this study, as study tasks added to the workload (e.g., completion of surveys) and introduced artificial timelines for some programs.

\section{Implications for Program Practices}

The findings of the current study suggest the significance of attending to change commitment and change efficacy within programs planning to undertake the QMS improvement process. As novice Affiliates and new mentoring programs are recruited to QMS, it will be important to assess why they are engaging in the initiative to understand their motivation and commitment to the process. Staff are more likely to engage fully when they are highly committed. As such, QMS should be framed as aligning with their program mission and values and should appeal to program needs (e.g., demonstrating quality to funders, recruiting mentors). Ensuring that staff are familiar with the EEPM and understand the value of implementing practice standards may also increase program commitment by helping them understand the importance of QMS processes. Offering incentives may help bring programs on board, but program staff still need to feel that they have some choice about QMS participation to be committed to its implementation. In addition, having strong interorganizational relationships (i.e., between MENTOR/expert Affiliates and novice Affiliates and between local Affiliate and 
mentoring programs) appears to help new Affiliate and program staff have trust in and commit to the QMS process. QMS leaders will need to invest in building these relationships as QMS expands nationwide, especially as they seek to engage programs that do not have established relationships with a local Affiliate.

Beyond being highly committed, novice Affiliate and mentoring program staff should feel they have the ability to be successful in implementing QMS. At the most basic level, staff need a clear understanding of what they are being asked to do and what resources and expertise will be needed for implementation in order to make an informed decision about whether to engage in the QMS process. In particular, novice Affiliates looking to build sustainable QMS processes, and programs implementing the initiative, need to ensure that staff responsible for QMS are supported by leadership and have sufficient time and expertise to devote to this work. Affiliates may also consider how to engage with external resources such as consultants or local nonprofits to supplement areas where they lack in-house expertise such as financial planning and program evaluation.

At the Affiliate level, assessing program commitment and efficacy can help staff identify programs that may not be ready to effectively engage in the QMS process and help programs determine what steps can be taken to prepare them to undergo this type of change effort. Helping programs reflect on their commitment and available resources can help programs considering QMS implementation to decide whether the timing is right for them. It can also help program leaders discern where to best allocate resources to support the change process. Programs that might appear to need QMS the most (e.g., new programs, programs with few recommended practices) may have lower levels of buy-in and fewer resources to support QMS. The findings suggest that preparing programs to engage in QMS by investing in increasing commitment and 
change efficacy prior to beginning the change process is an important step to support successful QMS implementation. Further, having access to the kind of support offered by the expert Affiliates, such as coaching and provision of materials, is critical as well.

Although these recommendations for practice focus on the specific example of QMS implementation, the general lessons learned here likely transfer to other youth development settings in which a structured quality improvement process is guided by external consultants using the QRIS approach. Early evaluations of this approach in the youth development arena have shown promising results (e.g., Sheldon et al, 2010; Spielberger et al., 2009; Smith et al., 2012), but research also indicates the importance of attention to the human and organizational factors affecting implementation such as those described in the current study (e.g., Baldwin et al., 2015; Devaney et al., 2012). The well-established literature on organizational change emphasizes the conditions and resources that facilitate implementation of new practices (Aarons et al., 2011; Weiner et al., 2020), and youth programs require both internal capacity as well as appropriate infrastructure and support to promote quality improvement efforts (Durlak \& DuPre, 2008; Smith et al., 2006). As an initial investigation of a QRIS initiative in the youth development context, the current study calls attention to factors that may be particularly relevant for nonprofit youth program providers to foster change commitment and change efficacy.

\section{Implications for Future Research}

Prospective studies that measure the ORC of programs prior to participation in a quality improvement intervention would help to quantify its contribution to successful implementation and change processes. It would also be important to have assessments of programs that decline opportunities to participate in QRIS processes to discern whether there are meaningful differences in ORC in these programs that have implications for future program recruitment, 
fostering of program readiness, and implementation of the improvement process. Also needed is a better understanding of how organizations, such as Affiliates, responsible for administering a QRIS process can identify specific ways programs can assess and enhance ORC prior to the start of the intervention. Future research also could examine the assumptions that respondents gave for participation. For example, do funders and volunteers really consider quality issues and value the stamp of approval gained through a quality improvement process? Understanding whether the anticipated value added for programs is realized is important to inform future efforts. 


\section{References}

Authors, 2018.

Aarons, G. A., Hurlburt, M., \& Horwitz, S. M. (2011). Advancing a conceptual model of evidence-based practice implementation in public service sectors. Administration and Policy in Mental Health and Mental Health Services Research, 38(1), 4-23. doi:

$10.1007 / \mathrm{s} 10488-010-0327-7$

Aarons, G.A., Sommerfeld, D.H., Hecht, D.B., Silovsky, J.F., \& Chaffin, M.J. (2009). The impact of evidence-based practice implementation and fidelity monitoring on staff turnover: Evidence for a protective effect. Journal of Consulting \& Clinical Psychology, 77, 2, 270-280. https://doi.org/10.1037/a0013223

Baldwin, C. K., Stromwall, K., \& Wilder, Q. (2015). Afterschool youth program design and structural quality: Implications for quality improvement. Child \& Youth Services, 36(3), 226-247. https://doi.org/10.1080/0145935X.2015.1046592

Baldwin, C. K., \& Wilder, Q. (2014). Inside quality: Examination of quality improvement processes in afterschool youth programs. Child \& Youth Services, 35(2), 152-168. https://doi.org/10.1080/0145935X.2014.924346

Boller, K., Paulsell, D., Grosso, P. D., Blair, R., Lundquist, E., Kassow, D. Z., Kim, R., \& Raikes, A. (2015). Impacts of a child care quality rating and improvement system on child care quality. Early Childhood Research Quarterly, 30, 306-315. https://doi.org/10.1016/j.ecresq.2014.10.001

Braun, V., \& Clarke, V. (2006). Using thematic analysis in psychology. Qualitative Research in Psychology, 3(2), 77-101. https://doi.org/10.1191/1478088706qp063oa 
Cannon, J.S., Zellman, G.L., Karoly, L.A., \& Schwartz, H.L. (2017). Quality rating and improvement systems for early care and education programs: Making the second generation better. Rand Corporation.

Cross, A. B., Gottfredson, D. C., Wilson, D. M., Rorie, M., \& Connell, N. (2010). Implementation quality and positive experiences in after-school programs. American Journal of Community Psychology, 45(3-4), 370-380. https://doi.org/10.1007/s10464-010$9295-\mathrm{z}$

Dattalo, M., Wise, M., Ford, J. H., Abramson, B., \& Mahoney, J. (2017). Essential resources for implementation and sustainability of evidence-based health promotion programs: A mixed methods multi-site case study. Journal of Community Health, 42(2), 358-368. https://doi.org/10.1007/s10900-016-0263-x

Devaney, E., Smith, C., \& Wong, K. (2012). Understanding the "how" of quality improvement: Lessons from the Rhode Island Program Quality Intervention. Afterschool Matters, 16, 110.

DuBois, D. L., Holloway, B. E., Valentine, J. C., \& Cooper, H. (2002). Effectiveness of mentoring programs for youth: A meta-analytic review. American Journal of Community Psychology, 30, 157-197. https://doi.org/10.1023/A:1014628810714

DuBois, D. L., Portillo, N., Rhodes, J. E., Silverthorn, N., \& Valentine, J. C. (2011). How effective are mentoring programs for youth? A systematic assessment of the evidence. Psychological Science in the Public Interest, 12, 57-91. https://doi.org/10.1177/1529100611414806

Durlak, J. A., \& DuPre, E. P. (2008). Implementation matters: A review of research on the influence of implementation on program outcomes and the factors affecting 
implementation. American Journal of Community Psychology, 41, 327-350.

https://doi.org/10.1007/s10464-008-9165-0

Farahnak, L. R., Ehrhart, M. G., Torres, E. M., \& Aarons, G. A. (2020). The influence of transformational leadership and leader attitudes on subordinate attitudes and implementation success. Journal of Leadership \& Organizational Studies, 27(1), 98-111. https://doi.org/10.1177/1548051818824529

Ford, J.K., Lauricella, T.K., Van Fossen, J.A., \& Riley, S.J. (2021). Creating energy for change: The role of changes in perceived leadership support on commitment to an organizational change initiative. Journal of Applied Behavioral Science, 57, 2, 153-173. https://doi.org/10.1177/0021886320907423

Garringer, M., Kupersmidt, J., Rhodes, J. E., Stelter, R., \& Tai, T. (2015). Elements of Effective Practice for Mentoring (4th Edition). MENTOR: The National Mentoring Partnership.

Garringer, M, McQuillin, S. \& McDaniel, H. (2017) Examining youth services across America: Findings from the 2016 National Program Survey. MENTOR: The National Mentoring Partnership.

Goffin, S. G., \& Barnett, W. S. (2015). Assessing QRIS as a change agent. Early Childhood Research Quarterly, 30, 179-182. https://doi.org/10.1016/j.ecresq.2014.08.005

Granger, R. C. (2010). Understanding and improving the effectiveness of after-school practice. American Journal of Community Psychology, 45(3-4), 441-446. https://doi.org/10.1007/s10464-010-9301-5

Hallam, R., Hooper, A., Bargreen, K., Buell, M., \& Han, M. (2017). A two-state study of family child care engagement in quality rating and improvement systems: A mixed-methods 
analysis. Early Education and Development, 28(6), 669-683.

https://doi.org/10.1080/10409289.2017.1303306

Holt, D. T., \& Vardaman, J. M. (2013). Toward a comprehensive understanding of readiness for change: The case for an expanded conceptualization. Journal of Change Management, 13(1), 9-18. https://doi.org/10.1080/14697017.2013.768426

Jenkins, J. M., Duer, J. K., \& Connors, M. (2021). Who participates in quality rating and improvement systems? Early Childhood Research Quarterly, 54, 219-227. https://doi.org/10.1016/j.ecresq.2020.09.005

Karcher, M. J., Kuperminc, G. P., Portwood, S. G., Sipe, C. L., \& Taylor, A. S. (2006). Mentoring programs: A framework to inform program development, research, and evaluation. Journal of Community Psychology, 34(6), 709-725. https://doi.org/10.1002/jcop.20125

Keller, T. E. (2007). Program staff in youth mentoring programs: Qualifications, training, and retention. In Jean Rhodes (Ed.), Research in Action. MENTOR/National Mentoring Partnership.

Kelly, P., Hegarty, J., Barry, J., Dyer, K.R., \& Horgan, A. (2017). A systematic review of the relationship between staff perceptions of organizational readiness to change and the process of innovation adoption in substance misuse treatment programs. Journal of Substance Abuse Treatment, 80, 6-25. https://doi.org/10.1016/j.jsat.2017.06.001

Kerman, B., Freundlich, M., Lee, J. M., \& Brenner, E. (2012). Learning while doing in the human services: Becoming a learning organization through organizational change. Administration in Social Work, 36, 234-257. https://doi.org/10.1080/03643107.2011.573061 
Komosa-Hawkins, K. (2010). Best practices in school-based mentoring programs for adolescents. Child \& Youth Services, 31(3-4), 121-137. https://doi.org/10.1080/0145935X.2009.524477

Kupersmidt, J. B., Stump, K. N., Stelter, R. L., \& Rhodes, J. E. (2017). Mentoring program practices as predictors of match longevity. Journal of Community Psychology, 45(5), 630 645. https://doi.org/10.1002/jcop.21883

Larson, R. W., \& Walker, K. C. (2010). Dilemmas of practice: Challenges to program quality encountered by youth program leaders. American Journal of Community Psychology, 45(3-4), 338-349. https://doi.org/10.1007/s10464-010-9307-z

Lengnick-Hall, R., Stadnick, N.A., Dickson, K.S., Moullin, J. C., Aarons, G. A. (2021). Forms and functions of bridging factors: specifying the dynamic links between outer and inner contexts during implementation and sustainment. Implementation Science, 16, 34. https://doi.org/10.1186/s13012-021-01099-y

Maynard, B.R. (2010). Social service organizations in the era of evidence-based practice: The learning organization as a guiding framework for bridging science to service. Journal of Social Work, 10, 3, 301-316. https://doi.org/10.1177/1468017309342520

McBeath, B., \& Hopkins, K. (2019) Navigating complex frontiers: Introduction to the special issue on "The future of human service organizational and management research", Human Service Organizations: Management, Leadership \& Governance, 43(4), 229-240. https://doi.org/10.1080/23303131.2019.1680023

Miles, M. B., Huberman, A. M., \& Saldana, J. (2014). Qualitative data analysis: A methods sourcebook (3rd ed.). Sage Publications. 
Miller, A. (2007). Best practices in formal youth mentoring. In T. D. Allen \& L. T. Eby (Eds.), Blackwell Handbook of Mentoring: A Multiple Perspectives Approach, (pp. 307-324). Oxford: Blackwell.

Mitchell, A. (2005). Stair steps to quality: A guide for states and communities developing quality rating systems for early care and education. United Way of America.

Moullin, J.C., Dickson, K.S., Stadnick, N.A., Rabin, B., \& Aarons, G. A. (2019). Systematic review of the Exploration, Preparation, Implementation, Sustainment (EPIS) framework. Implementation Science, 14(1). https://doi.org/10.1186/s13012-018-0842-6

Phelps, C., Heidl, R., \& Wadhwa, A. (2011). Knowledge, networks, and knowledge networks: A review and research agenda. Journal of Management, 38(4), 1115-1166. https://doi.org/10.1177/0149206311432640

Raposa, E. B., Dietz, N., \& Rhodes, J. E. (2017). Trends in volunteer mentoring in the United States: Analysis of a decade of census survey data. American Journal of Community Psychology, 59(1-2), 3-14. https://doi.org/10.1002/ajcp.12117

Raposa, E. B., Rhodes, J., Stams, G. J. J. M., Card, N., Burton, S., Schwartz, S., Sykes, L. A. Y., Kanchewa, S., Kupersmidt, J., \& Hussain, S. (2019). The effects of youth mentoring programs: A meta-analysis of outcome studies. Journal of Youth and Adolescence, 48, 423-443. https://doi.org/10.1007/s10964-019-00982-8

Rhodes, J. E., \& DuBois, D. L. (2008). Mentoring relationships and programs for youth. Current Directions in Psychological Science, 17(4), 254-258. https://doi.org/10.1111/j.14678721.2008.00585.x 
Sanclimenti, J., \& Caceda-Castro, L. E. (2017). Children's Bureau training and technical assistance: Synthesis of lessons learned from child welfare implementation projects. Training and Development in Human Services, 9, 34-40.

Sheldon, J., Arbreton, A., Hopkins, L., \& Grossman, J. B. (2010). Investing in success: Key strategies for building quality in after-school programs. American Journal of Community Psychology, 45(3-4), 394-404. https://doi.org/10.1007/s10464-010-9296-y

Sipe, C. L. (2002). Mentoring programs for adolescents: A research summary. Journal of Adolescent Health, 31(6), 251-260. https://doi.org/10.1016/S1054-139X(02)00498-6

Smith, C., \& Akiva, T. (2008). Quality accountability: Improving fidelity of broad developmentally focused interventions. In H. Yoshikawa \& B. Shinn (Eds.), Transforming social settings: Towards positive youth development (pp. 192-212). New York: Oxford University Press.

Smith, C., Akiva, T., Arrieux, D., \& Jones, M. M. (2006). Improving quality at the point of service. New Directions for Youth Development, 2006(112), 93-108. https://doi.org/10.1002/yd.195

Smith, C., Akiva, T., Sugar, S. A., Lo, Y., Frank, K. A., Peck, S. C., Cortina, K. S., \& Devaney, T. (2012). Continuous quality improvement in afterschool settings: Impact findings from the Youth Program Quality Intervention study. Washington, DC: Forum for Youth Investment.

Smith, C., Devaney, T. J., Akiva, T., \& Sugar, S. A. (2009). Quality and accountability in the out-of-school-time sector. New Directions for Youth Development, 2009(121), 109-127. https://doi.org/10.1002/yd.299 
Spielberger, J., Lockaby, T., Mayers, L., \& Guterman, K. (2009). Ready for Prime Time:

Executive summary: Implementing a formal afterschool quality improvement system by

Prime Time Palm Beach County. Chapin Hall at the University of Chicago.

Stoeger, H., Balestrini, D. P., \& Ziegler, A. (2021). Key issues in professionalizing mentoring practices. Annals of the New York Academy of Sciences, 1483, 5-18.

https://doi.org/10.5283/epub.44618

Tolan, P. H., Henry, D. B., Schoeny, M. S., Lovegrove, P., \& Nichols, E. (2014). Mentoring programs to affect delinquency and associated outcomes of youth at risk: A comprehensive meta-analytic review. Journal of Experimental Criminology, 10(2), 179-206.

https://doi.org/10.1007/s11292-013-9181-4

Tout, K. (2013). Look to the stars: Future directions for the evaluation of quality rating and improvement systems. Early Education \& Development, 24(1), 71-78.

https://doi.org/10.1080/10409289.2013.741912

Wandersman, A., Clary, E. G., Forbush, J., Weinberger, S. G., Coyne, S. M., \& Duffy, J. L. (2006). Community organizing and advocacy: Increasing the quality and quantity of mentoring programs. Journal of Community Psychology, 34(6), 781-799. https://doi.org/10.1002/jcop.20129

Weiner, B. J. (2009). A theory of organizational readiness for change. Implementation Science, 4(1), 67. https://doi.org/10.1186/1748-5908-4-67

Weiner, B. J., Amick, H., \& Lee, S. Y. D. (2008). Conceptualization and measurement of organizational readiness for change: A review of the literature in health services research and other fields. Medical Care Research and Review, 65(4), 379-436.

https://doi.org/10.1177/1077558708317802 
Weiner, B. J., Clary, A. S., Klaman, S. L., Turner, K., \& Alishahi-Tabriz, A. (2020).

Organizational readiness for change: What we know, what we think we know, and what we need to know. In Albers, B., Shlonsky, A., \& Mildon, R. (Eds), Implementation Science 3.0 (pp. 101-144). Springer.

Yohalem, N., Granger, R. C., \& Pittman, K. J. (2009). The quest for quality: Recent developments and future directions for the out-of-school-time field. New Directions for Youth Development, 2009(121), 129-140. https://doi.org/10.1002/yd.300

Yohalem, N., \& Wilson-Ahlstrom, A. (2010). Inside the black box: Assessing and improving quality in youth programs. American Journal of Community Psychology, 45(3-4), 350357. https://doi.org/10.1007/s10464-010-9311-3

Zellman, G. L., \& Perlman, M. (2008). Child-care quality rating and improvement systems in five pioneer states: Implementation issues and lessons learned. RAND Corporation. 
This is the author's accepted manuscript without copyediting, formatting, or final corrections. It will be published in its final form in an upcoming issue of the Journal of the Society for Social Work and Research, published by The University of Chicago Press on behalf of The Society for Social Work and Research. Include the DOI when citing or quoting: https://doi.org/10.1086/718516 Copyright 2021 The Society for Social Work and Research.

Table 1

State-level Affiliate and Mentoring Program Staff Characteristics $\left(n=43^{1}\right)$

\begin{tabular}{lc}
\hline Staff Characteristics & M(SD)/\% \\
\hline Age (range: 26-68 years) & $41.1(12.1)$ \\
Gender & \\
$\quad$ Female & 83.7 \\
$\quad$ Male & 16.3 \\
Racial Identity & \\
$\quad$ White & 55.8 \\
African-American & 27.9 \\
Multiracial & 9.3 \\
Other & 7.0 \\
Capacity & \\
Paid Staff & 90.7 \\
Volunteer & 9.3 \\
Years Working at Agency & \\
< 1 year & 7.1 \\
1-5 years & 57.1 \\
> 5-10 years & 21.4 \\
> 10 years & 14.3 \\
\hline
\end{tabular}

Note. ${ }^{1}$ Data were missing from 1 participant. 
This is the author's accepted manuscript without copyediting, formatting, or final corrections. It will be published in its final form in an upcoming issue of the Journal of the Society for Social Work and Research, published by The University of Chicago Press on behalf of The Society for Social Work and Research. Include the DOI when citing or quoting: https://doi.org/10.1086/718516 Copyright 2021 The Society for Social Work and Research.

Table 2

Mentoring Program Characteristics $\left(n=19^{1}\right)$

\begin{tabular}{|c|c|}
\hline Program Characteristics & $\%$ \\
\hline \multicolumn{2}{|l|}{ Organizational Structure } \\
\hline Embedded in Larger Non-Profit & 42.1 \\
\hline Independent Non-Profit Mentoring Program & 36.8 \\
\hline School or School District & 10.5 \\
\hline Other & 10.5 \\
\hline Nationally Affiliated & 15.8 \\
\hline \multicolumn{2}{|l|}{ Setting } \\
\hline Urban & 73.7 \\
\hline Suburban & 21.1 \\
\hline Rural & 5.2 \\
\hline \multicolumn{2}{|l|}{ Years in Operation } \\
\hline$<10$ years & 31.6 \\
\hline $10-20$ years & 36.8 \\
\hline $20+$ years & 31.6 \\
\hline \multicolumn{2}{|l|}{ Youth Served Annually } \\
\hline$<20$ & 26.3 \\
\hline $20-49$ & 21.2 \\
\hline $50-99$ & 15.8 \\
\hline $100+$ & 36.8 \\
\hline \multicolumn{2}{|l|}{ Model $^{2}$} \\
\hline One-on-one Only & 57.9 \\
\hline Combination (One-on-one and Either Group or Multi-mentor) & 36.8 \\
\hline Team & 5.3 \\
\hline
\end{tabular}

Note. ${ }^{1}$ Program characteristics were not provided by 1 mentoring program.

${ }^{2}$ Group mentoring is one mentor meeting with a group of mentees. Multi-mentor matches multiple mentors with one mentee. Team mentoring has multiple mentors matched with a group of mentees. 
This is the author's accepted manuscript without copyediting, formatting, or final corrections. It will be published in its final form in an upcoming issue of the Journal of the Society for Social Work and Research, published by The University of Chicago Press on behalf of The Society for Social Work and Research. Include the DOI when citing or quoting: https://doi.org/10.1086/718516 Copyright 2021 The Society for Social Work and Research.

Table 3

Summary of Themes Supporting Organizational Readiness for Change

\begin{tabular}{|c|c|c|c|}
\hline \multicolumn{2}{|c|}{ Commitment to the Change Process } & \multicolumn{2}{|c|}{ Change Efficacy } \\
\hline Affiliates & Mentoring Programs & Affiliates & Mentoring Programs \\
\hline Fit/expansion of QMS with Affiliate's & Value of EEPM standards & Planning process & Clear expectations for workload/process \\
\hline role/mission & Demonstrating/improving mentoring & Clear expectations for workload/process & Staff time \\
\hline Value of EEPM standards & program quality & Staff expertise & Staff stability \\
\hline Demonstrating/improving mentoring & Trusting relationship with Affiliate & Staff stability & Affiliate technical assistance \\
\hline program quality & Objectivity of outside opinion & Staff time & Fit of timing with program calendar \\
\hline Looking for QRIS process & Self-assessment opportunity & Funding & Organizational culture of learning \\
\hline Stakeholder support & Stakeholder support & Availability of existing QMS tools & \\
\hline & & Expert Affiliate support & \\
\hline & & Peer Affiliate support & \\
\hline
\end{tabular}

